\title{
Editorial
}

\section{Nutrition information and front-of-pack labelling: issues in effectiveness}

In this issue of Public Health Nutrition, eight papers present the latest research on how to display nutrition information for packaged foods and restaurant meals to encourage consumers to make healthier choices. These papers highlight the many factors that can influence effective communication of nutrition information to ensure that consumers notice, correctly interpret and utilize the information, as well as the difficulties in implementing voluntary $v$. mandatory nutrition reporting systems.

Research into the effectiveness of nutrition labels dates back as early as the $1970 \mathrm{~s}^{(1)}$, with major milestones being the introduction of various legislative pieces around the world, including the Nutrition Labeling and Education Act of 1990 (NLEA) in the USA and the Food Information to Consumers (FIC) Regulation 2011 in Europe. Certain aspects of nutrition labelling have become mandatory, for example the nutrition facts panel in the USA, a nutrition information panel in Australia (Food Standards Australia New Zealand 2011) or the nutrition table per $100 \mathrm{~g} / \mathrm{ml}$, on the back of a pack, in the EU (EU 1169/2011).

\section{Consumer awareness}

However, providing consumers with nutrition information is just the first step in encouraging consumers to make healthier choices. High awareness of the availability of nutrition information is also required. A review of recent research on the effectiveness of menu labelling indicates that at least $70 \%$ of consumers must notice the energy (calorie) postings on menus for menu labelling to affect restaurant purchases ${ }^{(2)}$. Nevertheless, the authors calculate an effect size of $325 \cdot 7-419 \cdot 5 \mathrm{~kJ}(77 \cdot 8-100 \cdot 2 \mathrm{kcal})$ reduction in their meta-analysis, which can be taken as a first indicator that menu labelling may offer the possibility of effectively reducing energy ordered and consumed away from home. They also suggest that increased consumer awareness and understanding of menu labelling, especially in the USA where such policies were first implemented, may help explain why the studies in their analysis - all conducted from 2012 to 2014 - were more likely to indicate effectiveness compared with earlier studies.

\section{Understanding and interpretation}

Understanding and correctly interpreting the nutrition information is another prerequisite, as highlighted by other papers in this issue. For example, Nyilasy et al. ${ }^{(3)}$ show that colour coding of information on the nutrition facts panel may even lead to adverse effects as the overall perception of a category as more $v$. less healthful can bias healthfulness evaluations of individual products, up to a point where healthy products are perceived as less healthy when the nutrition facts panel is colour-coded. Brand et $a l^{(4)}$ demonstrate in a series of studies that other information presented on food packaging (i.e. pictures of the product) can impact consumers' and even food professionals' understanding of appropriate serving sizes. A clear indication on the packaging as to which parts of the food are included in the nutrition label can help reduce this serving size bias.

\section{Differential use by consumers}

While there is evidence of an association between label use and diet quality, it is not clear how to achieve consistent label use across consumers, as highlighted in two additional studies. In their review of studies from the USA, Canada, the UK and South Korea, Christoph et al. ${ }^{(5)}$ present a weighted average of label use among college students and young adults of $36.5 \%$. About the same percentage use labels 'sometimes' and slightly less than one-third use them rarely or never. This certainly leaves room for improvement. One step forward in understanding factors affecting label use may be the findings of $\mathrm{An}^{(6)}$ who shows that (regular) label use is significantly higher among those who have been diagnosed with (pre-)diabetes, compared with those who either have undiagnosed (pre-)diabetes or no (pre-)diabetes at all. Such a disease diagnosis is just one of many examples of health goal activation, which has received much attention in recent consumer behaviour research ${ }^{(7)}$. Motivation is increasingly seen as the key factor in driving behavioural change towards a healthier lifestyle.

\section{Front-of-pack labelling}

Public health experts have also concluded that simply providing nutrition information without helping consumers interpret the information is unlikely to effectively encourage most consumers to make healthier choices ${ }^{(8)}$. As a result, public health organizations increasingly call for front-of-pack (FOP) labelling systems that quickly convey product healthfulness through simple, easy-to-understand visuals. 
A number of these systems have been proposed and tested. In a previous review of the literature on FOP label effectiveness, Hawley et $a l^{(9)}$ concluded that a Multiple Traffic Light approach, which highlights good (green), neutral (amber) and poor (red) levels of sugar, fat and sodium, is most effective at helping consumers identify healthier choices. Littlewood et al. ${ }^{(2)}$ also found that inclusion of traffic light and other interpretive information improves the effectiveness of menu labelling.

As a result, numerous public health organizations have proposed voluntary FOP systems to encourage consumers to make healthier choices. The Food Standards Agency recommends a traffic light approach for use in the UK. In the USA, the Institute of Medicine has recommended a three-star system, while the Australian Government Department of Health recommends a five-star Health Star Rating system. A plan to introduce a European nutrient profiling system to guide nutrition- and health-related information on pack has been on hold since 2010. In 2015, the WHO Regional Office for Europe published a nutrient profile model for adaption by EU Member States; however, this remains a suggestion and does not constitute a mandatory regulation.

Research into the effectiveness of nutrient profiles that form the basis for FOP labels is now timelier than ever. In this issue, Epstein et al. ${ }^{(10)}$ show that using NuVal ${ }^{\circledR}$ scores (a proprietary nutrient profiling system utilized by some manufacturers in the USA) to highlight the healthfulness of foods could lead to improved overall diet quality of supermarket products selected by study participants. In contrast, price changes, including subsidies for healthier foods and higher prices for nutrient-poor products, had no significant effect. Carrad et $a l .{ }^{(11)}$ found that both the Institute of Medicine three-star and the Australian Health Star Rating systems had good agreement in product healthfulness and that both systems rated products broadly in line with the Australian Dietary Guidelines, with core foods rated higher than discretionary ones.

\section{The limits of voluntary action by manufacturers and retailers}

Perhaps the greatest barrier to implementation of FOP labelling systems is that, despite widespread recommendations by the public health community for their use, these systems remain voluntary. Therefore, every manufacturer and every retailer is free to apply FOP label design in a way they see fit. Previous research has already argued that multiple FOP labels in the market can hinder consumer understanding and possibly discourage their use ${ }^{(12)}$. International comparisons showcase the need for a comprehensive approach to FOP labelling as the authors argue that voluntary schemes can lead to consumer confusion: has a product not received a star rating because it does not meet the nutritional requirements or because the manufacturer has decided not to participate in that programme? Furthermore, the authors call for a standardized serving size for food and drink categories to ensure consistent ratings across several systems.

The latest findings in this issue underline the need for mandatory action, with supermarkets in the UK employing anywhere from three to seven different nutrition label designs on their products sold online, including location on the page, inclusion of Reference Index values and the use of colour $^{(13)}$. Although retailers are required to provide the nutrition table for all products, there are no guidelines on placement and presentation for online retailers. Additionally, inconsistencies in food nutrition labelling and the use of FOP systems between the supermarkets and their online shops were reported, thus adding to the range of nutrition information that consumers are exposed to, even if they buy all their groceries within one chain. Notably, three retailers were more likely to use traffic light colours online than in their supermarkets.

Finally, industry may choose not to implement nutrition reporting and/or FOP systems at all when they are voluntary. The USA and some parts of Australia and Canada now require energy (calorie) labelling on restaurant menus and some other types of foods consumed out of home. However, these initiatives are voluntary in the UK and Ireland, and they are still only being discussed in other parts of the world, such as Denmark and Asia. As such, findings are diverse and often difficult to compare, as noted by Littlewood et $a l .^{(2)}$. Furthermore, there are numerous issues in implementing effective menu labelling and encouraging restaurants to participate, including how to calculate the nutritional values, for rapidly changing menus and exotic ingredients, with limited time and monetary resources and possible staff resistance ${ }^{(14,15)}$. These issues in menu labelling further highlight the difficulties of implementing effective voluntary nutrition reporting systems of any kind.

In sum, while we cannot yet offer a complete solution on food labels to guide healthy choices, this issue presents a selection of new research findings on the various aspects discussed above, reviews existing evidence and offers recommendations for future research.

Sophie Hieke ${ }^{1}$ and Jennifer L. Harris ${ }^{2}$

${ }^{1}$ Head of Consumer Insights European Food Information Council (EUFIC) Tassel House, Rue Paul-Emile Janson 6 1000 Brussels, Belgium

Email: sophie.hieke@eufic.org

${ }^{2}$ The Rudd Center for Food Policy \& Obesity University of Connecticut One Constitution Plaza, Suite 600 Hartford, CT 06103, USA Email: jennifer.harris@uconn.edu 


\section{References}

1. Hieke S \& Taylor C (2011) A critical review of the literature on nutritional labelling. $J$ Consum Aff $\mathbf{4 6}$, 120-156.

2. Littlewood JA, Lourenco S, Iversen CL et al. (2016) Menu labelling is effective in reducing energy ordered and consumed: a systematic review and meta-analysis of recent studies. Public Health Nutr 19, 2106-2121.

3. Nyilasy G, Lei J, Nagpal A et al. (2016) Colour correct: the interactive effects of food label nutrition colouring schemes and food category healthiness on health perceptions. Public Health Nutr 19, 2122-2127.

4. Brand J, Wansink B \& Cohen A (2016) Frosting on the cake: pictures on food packaging bias serving size. Public Health Nutr 19, 2128-2134.

5. Christoph MJ, An R \& Ellison B (2016) Correlates of nutrition label use among college students and young adults: a review. Public Health Nutr 19, 2135-2148.

6. An R (2016) Diabetes diagnosis and nutrition facts label use among US adults, 2005-2010. Public Health Nutr 19, 2149-2156

7. Van Herpen E \& van Trijp HCM (2011) Front-of-pack nutrition labels. Their effect on attention and choices when consumers have varying goals and time constraints. Appetite 57, 148-160
8. Institute of Medicine (2012) Front-of-Package Nutrition Rating Systems and Symbols: Promoting Healthier Choices. Washington, DC: The National Academies Press.

9. Hawley KL, Roberto CA, Bragg MA et al. (2013) The science on front-of-package food labels. Public Health Nutrition 16, 430-439.

10. Epstein LH, Finkelstein EA, Katz DL et al. (2016) Effects of nutrient profiling and price changes based on $\mathrm{NuVal}^{\circledR}$ scores on food purchasing in an online experimental supermarket. Public Health Nutr 19, 2157-2164.

11. Carrad AM, Loui JCY, Yeatman HR et al. (2016) A nutrient profiling assessment of packaged foods using two star-based front-of-pack labels. Public Health Nutr 19, 2165-2174.

12. Draper AK, Adamson AJ, Clegg S et al. (2013) Front-of-pack nutrition labelling: are multiple formats a problem for consumers? Eur J Public Health 23, 517-521.

13. Stones C (2016) Online food nutrition labelling in the UK: how consistent are supermarkets in their presentation of nutrition labels online? Public Health Nutr 19, 2175-2184.

14. Thomas E (2016) Food for thought: obstacles to menu labelling in restaurants and cafeterias (Commentary). Public Health Nutr 19, 2185-2189.

15. Nikolaou CK \& Lean MEJ (2016) Compulsory calorie labelling of foods. A response to 'Food for thought: obstacles to menu labelling in restaurants and cafeterias' by E Thomas (Letter to the Editor). Public Health Nutr 19, 2190-2191. 